\title{
Análisis de las acciones de juego en tenis de mesa a través de metodología observacional ${ }^{*}$
}

\author{
Mateo Nicolás Zamora-Quiroga** \\ Laura Andrea Ramírez-Sánchez ${ }^{* * *}$ \\ Juan Santiago Cortés-Cañón ${ }^{* * * *}$
}

Recibido: noviembre 11 del 2019 • Aceptado: febrero 24 del 2020

\section{Resumen}

El objetivo de este artículo se basa en describir y analizar los indicadores de las acciones de juego en deportistas de tenis de mesa, a partir de la metodología observacional con diversos componentes de categorización. Para ello, se grabaron tres partidos de cuatro deportistas de un club de tenis de mesa. Los partidos fueron grabados mediante videocámaras digitales para su posterior visualización y análisis. Los datos obtenidos para cada una de las categorías utilizadas fueron organizadas, almacenadas y cuantificadas en una base de datos. Se registró que la duración de los partidos fue $14.49 \pm 3.86$ distribuidos en $0.20 \pm 0.05$ pausa entre tantos y $0.31 \pm 0.06$ entre juegos. El número de golpeos totales por partido fue de $771.67 \pm 26.131$. Los resultados obtenidos indican que dichos componentes de categorización fueron fiables para la valoración

Artículo resultado de investigación. Financiación propia. Semillero de Psicología del deporte y el ejercicio usta. Universidad Santo Tomás. Bogotá, Colombia.

Citar como: Zamora-Quiroga, M., Ramírez-Sánchez, L. y Cortes-Cañón, J. (2020). Análisis de las acciones de juego en tenis de mesa a través de metodología observacional. Revista de Investigación Cuerpo, Cultura y Movimiento, 10(2), 19-43. DoI: https://doi.org/10.15332/2422474x/6224

** Estudiante de pregrado en Psicología, Universidad Santo Tomás, Bogotá, Colombia. Correo electrónico: mateozamora@usantotomas.edu.co. orcID: 0000-0002-6842-7380

*** Estudiante de pregrado en Psicología, Universidad Santo Tomás, Bogotá, Colombia. Correo electrónico: lauraaramirez@usantotomas.edu.co. oRCID: 0000-0001-5116-1873

**:* Estudiante de Cultura Física, Deporte y Recreación, Universidad Santo Tomás, Bogotá, Colombia. Correo electrónico: juan.cortesc@usantotomas.edu.co. oRCID: 0000-0003-3633-8057 
y descripción objetiva de las acciones de juego, así como su estructura temporal por cada participante. De igual forma, el estudio arrojó diferencias de las categorías de los jugadores evaluados, lo que nos permite dar cuenta del rendimiento individual de cada deportista basado en diferentes elementos que pueden ser mejorados en sus entrenamientos.

Palabras clave: Análisis notacional, deporte de raqueta, rendimiento deportivo, tiempo de juego. 


\title{
Analysis of Gaming Actions in Table Tennis Through an Observational Methodology
}

\begin{abstract}
This article aims to describe and analyze indicators of gaming actions in table tennis athletes, based on an observational methodology with various categorization components. For this purpose, 3 matches of 4 athletes from a table tennis club were recorded by means of digital video cameras for their later visualization and analysis. The data used for each of the categories were organized, stored, and quantified in a database. It was recorded that the duration of the matches was $14.49 \pm 3.86$, distributed in a $0.20 \pm 0.05$ pause between goals and a $0.31 \pm 0.06$ pause between games. The total number of strokes per game was $771.67 \pm 26,131$. The results indicate these categorization components were reliable for the evaluation and objective description of the game actions, as well as their temporal structure for each participant. Similarly, the results showed differences in the categories of the players evaluated, which accounts for the individual performance of each athlete based on different elements that can be improved in their training.
\end{abstract}

Keywords: notational analysis, racket sport, sport performance, match duration. 


\section{Introducción}

Existe gran variedad de investigaciones enfocadas en deportes que se ejecutan con raquetas. Algunos son el tenis, el squash o el bádminton, en los que se investiga acerca de la relevancia de distintos elementos como la estructura temporal, acciones motrices o patrones tácticos del juego, tal y como lo menciona Cabello y González-Badillo (2003).

No obstante, dentro del tenis de mesa se descubren pocos estudios que logren describir con precisión los indicadores de las distintas variables que califican el juego en esta disciplina. Según Djokic (2006, citado por Kondrič, Zagatto y Sekulić, 2013), existen algunas publicaciones realizadas que intentan describir la dinámica interna en el deporte, identificando subcategorías dentro de las variables como la técnica y la táctica como patrón básico de estudio.

Hughes (1995, citado por Pradas, Floría, González, Carrasco y Bataller, 2012) menciona que algunas investigaciones pretenden trazar el esbozo del tenis de mesa moderno; sin embargo, resulta muy complejo analizar este deporte en tiempo real debido a los múltiples desplazamientos motrices que ocurren en periodos limitados a gran rapidez. Por tanto, es importante crear una guía confiable de observación y recolección de algunos componentes específicos que permitan examinar, comparar, analizar y sistematizar los gestos de juego que ocurren en un encuentro de tenis de mesa.

Dentro de los métodos empleados para evaluar con validez y coherencia los elementos clave de la competición destaca el análisis notacional (Hughes, 1995, citado por Pradas et al., 2012). El uso de este plan de visualización y análisis produce la posibilidad de construir unas tablas que contienen las variables y categorías pertinentes para una indagación acerca de las acciones de juego del deporte. Según Pradas, Salvá, González y González (2015), esta indagación comprende la descomposición y la distinción de propiedades técnicas y provisionales que se producen en un campeonato para lograr una comprensión en cuanto a la dificultad y la potencia a las que son sometidos los deportistas. Por tanto, es importante medir y calcular los señalizadores externos que intervienen en el juego, para así generar un perfil de las acciones de juego en el deporte y, asimismo, planificar un entrenamiento óptimo para el deportista.

Los indicadores de carga externa, según Pradas et al. (2015), son aquellos que muestran la contribución física que soporta el jugador, y tienen distintas 
naturalezas; por tanto, y con fines para esta investigación, estos indicadores nos ayudan a medir los factores de tiempo, pausa y esfuerzo, así como las técnicas que se utilizan y el número total de golpeos.

Acerca de las acciones de juego, se pueden encontrar distintas definiciones; sin embargo, en el contexto de este proyecto se considera la propuesta de Reyes, Raimundi y Gómez (2012) de describir y cuantificar las variables de las acciones de juego como aplicación de la técnica, entre otros. A su vez, el rendimiento deportivo está directamente relacionado con las acciones de juego, proporcionando información relevante sobre el trabajo realizado por los deportistas durante la práctica.

Olmedilla, Ortega, Andreu y Ortín (2010) definieron el rendimiento como el resultado de toda la dedicación e inversión de diversos recursos para con su deporte. Naturalmente, estos resultados están relacionados con la calidad, frecuencia, intensidad, recuperación y otros aspectos importantes de la preparación deportiva, para un adecuado rendimiento que permita un mejor desarrollo de las acciones de juego.

Según Lees (2003, citado por Roque y Iturriaga 2011), los deportes de raqueta han tenido un aumento de fama y técnica en la última década; por tal motivo, generan gran atractivo para la comunidad científica, enfocándose en diferentes metodologías de análisis en distintas partes de formación de las modalidades.

El uso de tecnologías y herramientas de medición en el deporte ha tenido una gran relevancia en los estudios deportivos (Pradas et al., 2012; Castellano y Casamichana, 2014). Dicho interés es causado gracias a la importancia de construir un detallado análisis de las características de juego presentes en las disciplinas, en especial los de raqueta (bádminton, tenis, ping pong, pádel, squash y frontenis), debido a las numerosas situaciones motrices que suceden a altas velocidades y en cortos periodos de tiempo (Pradas et al., 2012).

Según Gómez (2014), el videoanálisis es una herramienta que logra adoptar las pautas necesarias para concebir una observación a gran detalle de partidos, independientemente del deporte que se busque analizar. Las tecnologías resultan ser instrumentos de observación y análisis que capturan y retienen información detallada y precisa de las situaciones motrices que se desean captar.

Por lo anterior, es posible la observación sistemática de las acciones de juego por medio de registro de los encuentros deportivos (Conejero-Suárez 
et al., 2017), permitiendo un análisis notacional que hace uso combinado de técnica, táctica y física (Pradas et al., 2012). En esta investigación el análisis de las acciones de juego se realizará por medio de la metodología observacional (Anguera y Mendo, 2015).

De esta forma, se han diseñado pocos sistemas de observación notacional; para ello, esta investigación tendrá algunos factores o categorías en cuenta, relacionados con el sistema o taxonomía de observación notacional propuesta por Pradas et al. (2012), que incluyen aspectos de carga externa y que miden la duración de los partidos, la pausa entre juegos o sets y la pausa entre tantos.

Como lo sostiene Martínez (2013), el análisis notacional presenta una gran importancia a la hora de realizar un proceso de feedback con el tenista. También se tiene en cuenta que en este análisis es posible recopilar elementos que dan cuenta del deportista de manera objetiva, algunos de los cuales son el análisis metódico, técnico y de acción.

Por otra parte, el cuestionamiento de esta investigación se plantea así: ¿Cuáles son las características de acciones de juego de cuatro tenistas de mesa a partir de un análisis situacional mediante la metodología observacional?

Dentro de la bibliografía científica siempre ha existido un interés en el desarrollo deportivo, en la descomposición de lo característico de grandes competiciones, siendo un aspecto de gran importancia en los diferentes deportes, incluidos los de raqueta (Cabello, 2000). Por esa razón, se han realizado diferentes estudios dentro de los deportes tales como son squash, bádminton e incluso tenis, investigando acerca de la organización de tiempo, las actividades motrices o los modelos tácticos del juego (Cabello y González-Badillo, 2003).

Kondrič et al. (2013) mencionan que existen algunas publicaciones en las que describen métodos no tan sofisticados que pretenden generar una respuesta sobre la dinámica interna que presenta el tenis de mesa, mediante la identificación de las variables técnicas y tácticas como principio o patrón básico para su estudio, teniendo en cuenta que la mayoría fueron realizadas antes de la profunda transformación que hizo la ITTF (International Table Tennis Federation) acerca del reglamento técnico del juego que tuvo lugar a finales de los años 90 e inicios del 2000. Las modificaciones han generado nuevos aspectos que han afectado la estructura y el desarrollo del juego, marcando un antes y después dentro del deporte (Takeuchi, Kobayashi, Hiruta y Yuza, 2002).

La aplicación de tecnologías novedosas para el análisis de la táctica en tenis, según Reid (2011), da cuenta de dos tipos de observaciones: 
una observación directa, relacionada con la aplicación durante el partido, y una observación indirecta, conectada con la interpretación de muestra audiovisuales.

Para Roque e Iturriaga (2007) es relevante que habitualmente, a la hora de realizar una observación desde el punto de vista del entrenador, existe un factor intrínseco que es el conocimiento en las situaciones que necesitan de análisis y evaluación, pero por otro lado existe un sesgo relacionado con los aspectos sensoriales; sin embargo, este tipo de técnica es sometido a una prueba de rigurosidad para que sea posible su uso.

Respecto a la técnica y táctica del tenis de mesa, Malagoli, Di Michele y Merni (2014) proponen las características de tiro en jugadores de tenis de mesa de alto rendimiento estableciendo una comparación entre los estilos de juegos de los jugadores. El estudio fue realizado por asiáticos y europeos, y se examinó el rebote de la pelota para los servicios, el tipo de golpe y los movimientos. Se pudo evidenciar que existe una diferencia entre el uso de los golpes y los movimientos entre unos y otros, destacando que los asiáticos usan golpes ofensivos y muestran mayor efectividad en el servicio y el contraataque.

Adicionalmente, existen algunas herramientas usadas para el desarrollo de la táctica en el tenis de campo. Por ejemplo, Sanz y Terroba (2012) indican el uso de sistemas de apuntes y registros usados en smartphones, con el objetivo de registrar estadísticas globales en los partidos; algunos programas encontrados son Protracker y Tennis trakker. El uso de sistemas de análisis de video se caracteriza por la asignación de etiquetas en los diferentes momentos del partido; así es posible mezclar un análisis cuantitativo y cualitativo del desempeño del partido. Algunos programas utilizados son Dartfish, Interplay sports y Sportscode gamebreaker. El uso de estadísticas se da por medio de programas como Tenis prob, en donde se analiza la probabilidad constante de anotar un punto. Finalmente está la minería de datos e inteligencia artificial, la cual descubre relaciones y patrones entre los datos recolectados, predice los resultados y patrones de movimiento.

Es importante generar un patrón de análisis fiable y una recolección de datos que posibilite observar y diferenciar las acciones de juego que tengan lugar en un encuentro. De igual forma, es importante detallar cuáles son los objetivos que se plantea el deportista para obtener la victoria; de este modo, el modelo de análisis se puede considerar como el análisis del rendimiento que ayuda a mejorar los procesos de entrenamiento, entendido como 
la investigación en competición y entrenamiento en diferentes contextos (Gómez, 2017).

Teniendo en cuenta lo anterior, el planteamiento de objetivos en los deportistas está basado en establecer metas SMART que contengan un conjunto de elementos que hacen que se establezca una meta eficaz. Las metas SMART son específicas, cuantificables, orientadas a la acción, realistas, con factor de tiempo y autodeterminadas (Weinberg y Gould, 2010).

Existen varios métodos para la evaluación del deporte, aunque el más utilizado de forma válida y coherente para el tenis de mesa es el análisis notacional (Hughes, Hughes y Behan, 2007), basado en la observación, y que ayudará a hacer una evaluación de las acciones de juego que ejecutan los deportistas en su hábitat deportivo. En este orden de ideas, es importante tener en cuenta estos métodos de evaluación, ya que permitirá determinar la fiabilidad y la precisión a la hora de describir o mencionar el perfil de un deportista en relación con sus acciones de juego.

\section{Metodología}

Este proyecto de investigación se desarrolla bajo el enfoque cuantitativo, en el que se busca medir de manera objetiva las acciones de juego de cada tenista de mesa. La presente investigación se ubica en un alcance descriptivo y correlacional, usando un diseño de carácter no experimental longitudinal; con base en Abreu (2012), se recopilan los datos que describen los acontecimientos para su posterior organización y tabulación. Esto quiere decir que se medirá la variable de las acciones de juego, realizándose en tres momentos por participante, con una semana entre competencias. El análisis de las acciones de juego en jugadores de competencia se aborda desde un alcance descriptivo que genera medidas de tendencia central y de dispersión.

\section{Población}

La población se compone por un conjunto de jugadores de tenis de mesa perteneciente al club R y E. Dichos jugadores pertenecen a los primeros 10 lugares en los rankings mensuales del club. 


\section{Muestra}

Los participantes de este estudio fueron cuatro deportistas del Club de tenis de mesa $\mathrm{R}$ y E, seleccionados bajo un muestreo no probabilístico. Según Hernández, Fernández y Baptista (2014), un muestreo no probabilístico es aquel cuya elección de elementos no depende de la probabilidad, sino de las causas relacionadas con la investigación; de igual forma, la muestra estará compuesta por participantes voluntarios.

El criterio de inclusión contiene participantes mayores de edad con un lapso de participación activa de los rankings superior a dos meses y que practiquen el deporte con una intensidad de al menos tres veces a la semana. Además, el criterio de responsabilidad consistió en el diligenciamiento del consentimiento informado.

\section{Instrumentos y herramientas}

En la elaboración de este estudio, se contó con la taxonomía de las acciones de juego propuesta por Pradas et al. (2012). Se diseñó un formato compuesto por un sistema de observación notacional que comprende las siguientes categorías: duración de partidos, pausa entre tantos, pausa entre juegos, tantos totales, tantos por set, golpes totales, técnica drive, técnica de revés; cada una de estas técnicas estuvieron especificadas por las acciones de juego topspin, lift o nimb, cortada, saque y sin efecto. Todos los elementos del formato de campo son de tipo cerrado y codificación única.

\section{Procedimiento}

\section{Fase 1}

Se socializaron las instrucciones a los deportistas escogidos como participantes del estudio, seguido de la firma del consentimiento informado; posteriormente, se llevó a cabo la grabación de los partidos para el posterior análisis de las acciones de juego, teniendo en cuenta los criterios de inclusión. Para ello, se utilizaron dos cámaras Cannon T5I, alejadas de la mesa con una distancia de tres metros de su lateral y elevadas Berston BST 3560 a dos metros de altura. 
Para la grabación, la mesa de juego se dividió en dos mitades independientes, cada una con una cámara, obteniéndose dos registros temporalmente iguales.

\section{Fase 2}

Se analizaron los videos para proceder a su sincronización y, posteriormente, para el análisis notacional. Para ello, se utilizó el programa Adobe Premiere Pro, que permitió la sincronización de los videos y la visualización de las acciones de juego fotograma por fotograma. Finalmente, se completó el cuadro con las categorías y las taxonomías correspondientes a la tabla propuesta por Pradas et al. (2012).

\section{Fase 3}

Se hizo la retroalimentación correspondiente a los deportistas que accedieron a ser parte de la investigación en la creación del perfil de las acciones de juego en tenis de mesa.

\section{Resultados y discusión}

Las edades de los participantes comprenden desde los 21 hasta los 48 años, todos de sexo masculino. El tiempo de práctica corresponde al tiempo en el que el participante inició una práctica concurrente del tenis de mesa y solo uno de los participantes presenta un nivel académico de estudiante universitario. Los datos personales, académicos y deportivos de los participantes se encuentran en la tabla 1.

Tabla 1. Datos personales y deportivos de los participantes

\begin{tabular}{ccclc}
\hline Participante & Edad & \multicolumn{1}{c}{ Sexo } & Tiempo de práctica & Nivel académico \\
\hline 1 & 21 años & Masculino & Hace 2 años & Estudiante \\
2 & 48 años & Masculino & Hace 8 años & Profesional \\
3 & 34 años & Masculino & Hace 9 años & Profesional \\
4 & 44 años & Masculino & Hace 20 años & Profesional \\
\hline
\end{tabular}

Fuente: elaboración propia. 
Los valores de carga externa correspondientes a la estructura temporal muestran la categoría "duración del partido", que se divide directamente en la "pausa entre tantos" y la "pausa entre juegos". La primera de ellas corresponde al tiempo que transcurre entre el tanto realizado y el punto de inicio del siguiente tanto; el segundo corresponde al tiempo que transcurre desde el tanto final del juego realizado y el inicio del primer tanto del próximo juego. A partir de los resultados generados por cada uno de los participantes, el total de los valores en la estructura temporal correspondientes a la carga externa indican que la duración de los partidos fue de $14.49 \pm 3.86$ min con un tiempo de pausa entre tantos de $0.20 \pm 0.05 \mathrm{~min}$ y de un tiempo de pausa entre juegos de $0.31 \pm 0.06 \mathrm{~min}$. Los demás valores correspondientes a la carga externa de la estructura temporal se muestran en la tabla 2.

En la carga externa correspondiente a las acciones de juego se encuentran los tantos realizados en cada uno de los juegos disputados por los participantes. La categoría "golpes totales" se dividen en "técnica de drive" y "técnica de revés", las cuales corresponden a los golpes totales realizados con las técnicas que se describen anteriormente. Los valores de los tantos disputados por juego y las técnicas realizadas por los participantes que hacen parte de la carga externa correspondiente a las acciones de juego se presentan en la tabla 3.

Dentro de la carga externa correspondiente a las acciones de juego de la técnica de drive, se encuentran cinco categorías que corresponden a los tipos de golpe de la técnica descrita anteriormente. En ella el participante 1 predomina en el golpe de cortada sobre los otros participantes en $40.33 \pm 8.74$; el participante 2 domina el golpe topspin un $33 \pm 7$; el participante 3 domina el golpe topspin en $50.67 \pm 37.65$ y el saque en $40.33 \pm 13.01$, y el participante 4 domina el golpe sin efecto en $29.67 \pm 9.62$. Los valores restantes que hacen parte a estas acciones de juego se muestran en la tabla 4. 
Tabla 2. Valor de carga externa (estructura temporal)

\begin{tabular}{|c|c|c|c|c|c|c|c|c|c|c|c|c|c|c|c|}
\hline & \multicolumn{3}{|c|}{ Participante 1} & \multicolumn{3}{|c|}{ Participante 2} & \multicolumn{3}{|c|}{ Participante 3} & \multicolumn{3}{|c|}{ Participante 4} & \multicolumn{3}{|c|}{ Total } \\
\hline & Media & DT & Rango & Media & DT & Rango & Media & DT & Rango & Media & DT & Rango & Media & DT & Rango \\
\hline Duración de partido & 15.91 & 5.03 & $10.53-20.5$ & 10.32 & 1.79 & $8.43-10.53$ & 15.90 & 4.29 & $15.2-20.5$ & 15.84 & 4.31 & $12-20.5$ & 14.49 & 3.86 & $8.43-20.5$ \\
\hline Pausa entre tantos & 0.43 & 0.07 & $0.03-0.28$ & 0.12 & 0.02 & $0.03-0.82$ & 0.13 & 0.02 & $0.03-0.82$ & 0.11 & 0.05 & $0.03-0.42$ & 0.20 & 0.05 & $0.03-0.82$ \\
\hline Pausa entre juegos & 0.11 & 0.04 & $0.32-0.52$ & 0.39 & 0.06 & $0.33-0.45$ & 0.38 & 0.07 & $0.30-0.44$ & 0.38 & 0.07 & $0.21-0.52$ & 0.31 & 0.06 & $0.21-0.52$ \\
\hline
\end{tabular}

Fuente: elaboración propia con base en Pradas et al. (2015) 
Tabla 3. Valor de carga externa (acciones de juego)

\begin{tabular}{|c|c|c|c|c|c|c|c|c|c|c|c|c|}
\hline & \multicolumn{3}{|c|}{ Participante 1} & \multicolumn{3}{|c|}{ Participante 2} & \multicolumn{3}{|c|}{ Participante 3} & \multicolumn{3}{|c|}{ Participante 4} \\
\hline & Media & DT & Rango & Media & DT & Rango & Media & DT & Rango & Media & DT & Rango \\
\hline Tantos totales & 79.67 & 24.99 & $58-107$ & 55.00 & 1.41 & $54-58$ & 80.33 & 26.50 & $54-107$ & 68.00 & 16.97 & $56-80$ \\
\hline Tantos de Juego 1 & 8.00 & 2.65 & $6-11$ & 19.66 & 2.82 & $19-22$ & 5.50 & 2.52 & $5-10$ & 10.00 & 1.73 & $8-11$ \\
\hline Tantos de juego 2 & 9.67 & 1.53 & $8-11$ & 19.00 & 4.35 & $16-24$ & 10.00 & 4.36 & $5-10$ & 9.33 & 2.89 & $6-11$ \\
\hline Tantos de juego 3 & 9.00 & 3.46 & $5-11$ & 17.33 & 2.30 & $16-20$ & 6.00 & 2.65 & $5-10$ & 8.67 & 3.51 & $5-12$ \\
\hline Tantos de juego 4 & 9.00 & 1.41 & $8-10$ & - & - & - & 6.00 & 5.57 & $7-11$ & 11.50 & 0.71 & $11-12$ \\
\hline Tantos de Juego 5 & 15.00 & - & 15 & - & - & - & 4.33 & 7.51 & 13 & - & - & - \\
\hline Golpes totales & 246.00 & 62.10 & $186-310$ & 155.33 & 37.75 & $114-188$ & 185.67 & 108.67 & 104-309 & 184.67 & 52.79 & $147-245$ \\
\hline Técnicas de drive & 112.33 & 27.47 & $90-143$ & 97.00 & 23.64 & $72-119$ & 143.33 & 84.88 & $74-238$ & 100.00 & 24.58 & $82-128$ \\
\hline Técnicas de revés & 133.67 & 35.70 & $96-167$ & 58.33 & 14.36 & $42-69$ & 42.33 & 24.91 & $26-71$ & 84.67 & 28.22 & $65-117$ \\
\hline
\end{tabular}

Fuente: elaboración propia con base en Pradas et al. (2015) 
Tabla 4. Valores de carga externa (acciones de juego drive)

\begin{tabular}{|c|c|c|c|c|c|c|c|c|c|c|c|c|}
\hline & \multicolumn{3}{|c|}{ Participante 1} & \multicolumn{3}{|c|}{ Participante 2} & \multicolumn{3}{|c|}{ Participante 3} & \multicolumn{3}{|c|}{ Participante 4} \\
\hline & Media & DT & Rango & Media & DT & Rango & Media & DT & Rango & Media & DT & Rango \\
\hline Topspin & 12.33 & 3.06 & $9-15$ & 33.00 & 7.00 & $28-41$ & 50.67 & 37.65 & $26-94$ & 24.67 & 6.03 & $19-31$ \\
\hline Lift & 0.67 & 1.15 & $0-2$ & 8.33 & 3.78 & $4-11$ & 7.00 & 5.57 & $1-12$ & 2.00 & 3.46 & $0-6$ \\
\hline Cortada & 40.33 & 8.74 & $33-55$ & 11.33 & 7.02 & $4-18$ & 25.00 & 23.39 & $11-52$ & 16.00 & 11.27 & $9-29$ \\
\hline Saque & 37.33 & 13.58 & $29-53$ & 27.00 & 1.73 & $26-29$ & 40.33 & 13.01 & $27-53$ & 34.00 & 7.00 & $26-39$ \\
\hline Sin efecto & 21.67 & 3.06 & $19-25$ & 17.33 & 8.32 & $8-24$ & 20.33 & 16.07 & $2-32$ & 29.67 & 9.62 & $10-31$ \\
\hline
\end{tabular}

Fuente: elaboración propia con base en Pradas et al. (2015) 
En la carga externa que corresponde a las acciones de juego de la técnica de revés, al igual que en la técnica de drive, se describen cinco tipos de golpes que caracterizan esta técnica del tenis de mesa. De los tipos de golpe, el participante 1 predomina el golpe de sin efecto con $52.67 \pm 19.22$, y el golpe de cortada en $23 \pm 3.61$ y topspin en $43.33 \pm 12.01$; en el participante 2 se destaca el golpe de cortada en un $19.33 \pm 14.04$; en el participante 3 predominan las acciones sin efecto en $22 \pm 29.10 \mathrm{y}$, finalmente, el participante 4 usa más en el partido las acciones de topspin en $38.33 \pm 2.89$ del total de los golpes con técnica de drive. Los valores restantes que corresponden a esta carga externa se muestran en la tabla 5 .

El valor de la carga externa de los participantes que corresponde a las acciones de juego muestran que se hicieron un total $283 \pm 69.85$ de tantos, que se distribuyen en $43.16 \pm 9.72$ tantos en el juego 1 ; con $48 \pm 13.13$ tantos en el juego 2 ; con $41 \pm 11.92$ tantos en el juego 3 ; con $26.5 \pm 7.69$ tantos en el juego 4 y con $19.33 \pm 7.51$ tantos en el juego 5 . Los valores restantes se muestran en la tabla 6 .

Los golpes totales realizados se distribuyen en golpes con la técnica de $d r i$ ve y la técnica de revés. Se identificaron cinco golpes diferentes realizados para un total de $452.66 \pm 160.57$ golpes con la técnica de drive, que se distribuyen en $120.67 \pm 53.74$ golpes topspin, con $18 \pm 13.96$ golpes lift, $92.66 \pm 50.42$ golpes de cortada, otros $92.66 \pm 50.42$ golpes de saque y con $88.99 \pm 37.07$ golpes sin efecto. Los valores restantes se muestran en la tabla 7 .

Se realizó un total de $319 \pm 103.19$ golpes con la técnica de revés, que se distribuyen en $100.32 \pm 21.89$ golpes topspin, $31.99 \pm 17,96$ golpes nimb, $52.99 \pm 21.26$ golpes de cortada, $1.42 \pm 2.45$ golpes de saque y $128 \pm 84.13$ golpes sin efecto. Los demás valores correspondientes a la carga externa correspondiente a las acciones de juego de la técnica de revés se muestran en la tabla 8 . 
Tabla 5. Valores de carga externa (Acciones de juego revés)

\begin{tabular}{|c|c|c|c|c|c|c|c|c|c|c|c|c|}
\hline & \multicolumn{3}{|c|}{ Participante 1} & \multicolumn{3}{|c|}{ Participante 2} & \multicolumn{3}{|c|}{ Participante 3} & \multicolumn{3}{|c|}{ Participante 4} \\
\hline & Media & DT & Rango & Media & DT & Rango & Media & DT & Rango & Media & DT & Rango \\
\hline Topspin & 43.33 & 12.01 & $31-55$ & 10.33 & 3.78 & $6-13$ & 8.33 & 3.21 & $6-12$ & 38.33 & 2.89 & $35-40$ \\
\hline $\mathrm{Nimb}$ & 12.33 & 2.31 & $11-15$ & 5.66 & 1.52 & $4-7$ & 3.67 & 5.51 & $0-10$ & 10.33 & 8.62 & $1-18$ \\
\hline Cortada & 23.00 & 3.61 & $20-27$ & 19.33 & 14.04 & $6-34$ & 8.33 & 1.53 & $7-10$ & 2.33 & 2.08 & $0-4$ \\
\hline Saque & 2.33 & 4.04 & $0-7$ & 2.66 & 4.61 & $0-8$ & - & - & - & 0.67 & 1.15 & $0-2$ \\
\hline Sin efecto & 52.67 & 19.22 & $32-70$ & 20.33 & 8.96 & $10-26$ & 22.00 & 29.10 & $0-55$ & 33.00 & 26.85 & $17-64$ \\
\hline
\end{tabular}

Fuente: elaboración propia con base en Pradas et al. (2015) 
Tabla 6. Valor de carga externa del total de los participantes (acciones de juego)

\begin{tabular}{cccc}
\hline Categoría & Media & DT & Rango \\
\hline Tantos totales & 283.00 & 69.87 & $54-107$ \\
Tantos de Juego1 & 43.16 & 9.72 & $5-22$ \\
Tantos de juego2 & 48.00 & 13.13 & $5-24$ \\
Tantos de juego 3 & 41.00 & 11.92 & $5-20$ \\
Tantos de juego 4 & 26.50 & 7.69 & $7-12$ \\
Tantos de Juego 5 & 19.33 & 7.51 & $13-15$ \\
Golpes totales & 771.67 & 261.31 & $104-310$ \\
Técnicas de drive & 452.66 & 160.57 & $72-238$ \\
Técnicas de revés & 319.00 & 103.19 & $26-167$ \\
\hline
\end{tabular}

Fuente: elaboración propia con base en Pradas et al. (2015)

Tabla 7. Valores de carga externa del total de los participantes (acción de juego drive)

\begin{tabular}{cccc}
\hline Categoría & Media & DT & Rango \\
\hline Topspin & 120.67 & 53.74 & $9-94$ \\
Lift & 18.00 & 13.96 & $0-12$ \\
Cortada & 92.66 & 50.42 & $8-55$ \\
Saque & 138.66 & 35.32 & $26-53$ \\
Sin efecto & 88.99 & 37.07 & $2-32$ \\
\hline
\end{tabular}

Fuente: elaboración propia con base en Pradas et al. (2015) 
Tabla 8. Valores de carga externa total de los participantes (acción de juego revés)

\begin{tabular}{cccc}
\cline { 2 - 4 } & Media & DT & Rango \\
\hline Topspin & 100.32 & 21.89 & $6-55$ \\
Nimb & 31.99 & 17.96 & $0-18$ \\
Cortada & 52.99 & 21.26 & $0-34$ \\
Saque & 1.42 & 2.45 & $0-8$ \\
Sin efecto & 128.00 & 84.13 & $0-70$ \\
\hline
\end{tabular}

Fuente: elaboración propia con base en Pradas et al. (2015)

Principalmente la finalidad de esta investigación fue determinar las características de acciones de juego de cuatro tenistas de mesa a partir de un análisis situacional mediante la metodología observacional. Lo que se obtuvo en esta investigación fue que, en cuanto a los valores de carga externa, referido más a la estructura temporal, las medias en las duraciones del partido fueron de $14.49 \pm 3.86$ en comparación con los resultados de valor de carga externa referidos a la estructura temporal encontrados por Pradas et al. (2015), que fue de $37.7 \pm 15.6$. De esto se puede inferir que la duración de los partidos de los resultados de Pradas et al. (2015) es mayor en comparación con los resultados de la presente investigación.

Además, en otro estudio propuesto por Pradas, Pinilla, Quintas y Castellar (2014), con base en la comparación de valores de carga externa entre hombres y mujeres, los resultados de los hombres arrojan golpeos por partido de $475.2 \pm 146.1$ en partidos a 7 puntos, siendo estos un poco más de la mitad comparándolos con los resultados obtenidos de esta investigación con $771.67 \pm 261.31$ de golpeos totales por partido. De igual forma, es importante mencionar que es un poco difícil contrastar los resultados encontrados con otras investigaciones, ya que una variable importante - la cantidad de tantos- no es tenida en cuenta.

De acuerdo con las taxonomías propuestas para desarrollar un análisis de observación notacional, se definió que la taxonomía más completa encontrada fue la que propuso Prada et al. (2015), ya que permite analizar de una forma cómoda y clara las acciones de juego desarrolladas por el deportista, en comparación con otras taxonomías, por ejemplo, la que propone Sáez-Morales, 
Ruano-Anoceto, Gutiérrez-Pairol y Águila-Crespo (2018). Si bien esta última taxonomía presenta las acciones divididas en acciones ofensivas y defensivas, abriendo espacio a jugadores de estilo defensivo, al momento de analizar un partido, si un deportista de estilo ofensivo llega a generar acciones de juego de un jugador de defensa, podrían generar confusiones a la hora del análisis de la observación notacional; de igual forma, para el análisis notacional de esta taxonomía requeriría ser un experto en el estilo de juego para deportistas defensivos.

Teniendo en cuenta lo anterior, podemos decir que los análisis de las observaciones notacionales son muy eficaces a la hora de dar cuenta de la descripción de un dato, ya que permite tener una mayor precisión y seguridad a la hora de definir el comportamiento interno de algún deporte. Por ejemplo, en paralelo al tenis de mesa, existen deportes similares como el frontenis, de modo que, según Roque e Iturriaga (2011), la observación notacional permite analizar de forma objetiva variables como el gesto técnico de golpeo o efecto dado a la pelota, espacio de envío a la pared frontal, secuencia espacial más repetida, entre otras.

Adicionalmente, se han hecho análisis notacionales en deportes como voleibol (Medina, García, Morante y Rodríguez 2016; García, 2014), tenis (Varas y Gómez, 2016; Martínez, 2015; Reid, 2011) y baloncesto (Fernández, Camerino, Anguera y Jonsson, 2009; Fernández, Sánchez, Jiménez, Navarro y Anguera, 2012).

Cabe destacar que se cuenta con gran cantidad de programas que se usan en la metodología observacional como Protracker, Tennis trakker, Dartfish, Interplay sports y Sportscode gamebreaker (Sanz y Terroba, 2012); sin embargo, muchos de estos programas o software están diseñados para deportes de conjunto como lo es el fútbol, voleibol, entre otros, mientras que en los deportes que son más de carácter individual, muchas veces los programas diseñados son específicamente para ciertos tipos de deporte y muchas veces no logran dar respuesta sobre la dinámica interna del deporte en las que se identifiquen variables técnicas y tácticas, más aún en el tenis de mesa (Pradas et al., 2012).

De igual forma, hay que comprender que los análisis de las acciones de juego ayudan al mejoramiento del rendimiento. Según Sáez-Morales et al. (2018), el buen rendimiento está asociado al tiempo de entrenamiento. Teniendo en cuenta lo anterior, y añadiendo los análisis de las observaciones 
notacionales, podemos decir que dichos análisis pueden perfeccionar los tiempos de entrenamientos potenciando las virtudes del deportista y mejorando una habilidad, lo que reduce los márgenes de error en su ejecución; de esta forma, al mejorar los entrenamientos, podríamos decir que el rendimiento del deportista mejorará.

Asimismo, un estudio realizado por Rodríguez-Salazar y Montoya (2006) consistió en determinar si existen diferencias significativas en grupos entre pretest y postest para dos grupos experimentales, mas no para el grupo de control. Es decir, los deportistas que obtuvieron un programa de entrenamiento enfocados en ciertos factores como la atención lograron mejorar su desempeño deportivo significativamente, en comparación con el desempeño de aquellos que no tuvieron ningún tipo de entrenamiento.

Las investigaciones en deportes de gran complejidad como el tenis de mesa requieren de herramientas de evaluación objetivas y fiables para que se logre detallar y explicar de forma precisa el juego (Lupo, Tessitore, Minganti y Capranica, 2010, citado por Pradas et al., 2012).

Por medio de la metodología observacional, es posible establecer que los participantes tienen mejor rendimiento, ya que es viable realizar una retroalimentación bastante completa de los movimientos, de las acciones de juego y de los factores de carga externa, así como lo afirma Martínez (2013).

Para futuras investigaciones se recomienda grabar con más cámaras, con el fin de obtener una mejor perspectiva de visibilidad de la mesa, tener mayor cantidad de datos y establecer un perfil de rendimiento más completo. Asimismo, se recomienda el uso de software como Adobe Premiere usado en la investigación de Prada et al. (2012), pues facilita y complementa la sincronización de los datos y las etiquetas de las categorías que se usarán.

Igualmente, se podría plantear una mayor cantidad de grabaciones para así establecer mejores patrones de relación entre los datos encontrados. Adicionalmente, haciendo referencia a las categorías notacionales, se sugiere utilizar en su totalidad el modelo establecido por Prada et al. (2015) e incluir las categorías de carga externa en cuanto a la estructura temporal tiempo total de actividad y tiempo total de pausa, así como en cuanto a valores de carga externa de acciones de juego los desplazamientos. 


\section{Conclusiones}

Es posible hacer un análisis preciso y fiable de las acciones de juego en un partido de tenis de mesa, añadiendo a esto la estructura temporal del juego. Dicho análisis muestra que la duración total de los partidos es similar. Sin embargo, se pueden encontrar ciertas variaciones en cuanto al uso de golpes de revés y golpes de drive, es decir, hubo una gran predominancia en el golpe de drive en los participantes a excepción del participante 1. De esta forma, los resultados hallados en este estudio indican que el sistema notacional diseñado por Pradas et al. (2012) permite evaluar de forma objetiva y cuantificable las acciones desarrolladas durante el juego.

Obtener esta información implica una gran relevancia para el deportista y el entrenador, ya que puede ser considerada una pieza fundamental para la optimización del rendimiento deportivo. De igual forma, ayuda a proporcionar una retroalimentación inmediata sobre las acciones de juego del deportista para desarrollar estrategias más adecuadas en cuanto al entrenamiento.

La taxonomía y categorización empleada proporciona información importante y precisa acerca de la estructura temporal y acciones del juego, ya que puede dar cuenta del posible rendimiento deportivo. Por lo tanto, para un mejor rendimiento deportivo es necesario la aplicación de habilidades psicológicas tales como el establecimiento de objetivos, entre otros.

\section{Agradecimientos}

Al culminar este trabajo de investigación nos permitimos hacer llegar nuestros más sinceros agradecimientos a la Universidad Santo Tomás, la cual abrió sus puertas para formarnos profesionalmente con el fin de prestar un servicio comunidad por medio de sus excelentes docentes y de su ardua labor.

Agradecimientos al docente Fredy Rodríguez por su constante compañía y asesoramiento a lo largo del desarrollo del proyecto.

A los deportistas del Club R y E quienes se prestaron para el desarrollo de la investigación y quienes lograron brindar óptimamente los resultados gracias a sus habilidades y conocimiento en el deporte. 


\section{Referencias}

Abreu, J. (2012). Hipótesis, método \& diseño de investigación. Daena: International Journal of Good Conscience, 7(2), 187-197. Recuperado de http://www.spentamexico.org/v7-n2/7(2)187-197.pdf

Anguera, M. T. y Mendo, A. H. (2015). Técnicas de análisis en estudios observacionales en ciencias del deporte. Cuadernos de psicología del deporte, 15(1), 13-30. DOI: http://dx.doi.org/10.4321/S1578-84232015000100002

Castellano, J., y Casamichana, D. (2014). Deporte con dispositivos de posicionamiento global (GPS): Aplicaciones y limitaciones. Revista de Psicología del Deporte, 23(2), 0355-364. Recuperado de https://ddd.uab.cat/record/119442.

Cabello, D. (2000). Análisis de las características del juego en el bádminton de competición. Su aplicación al entrenamiento (Tesis Doctoral). Universidad de Granada, Granada.

Cabello, D. y González-Badillo, J. (2003). Analysis of the characteristics of competitive badminton. British Journal of Sports Medicine, 37(1), 62-66. DoI: https:// doi.org/10.1136/bjsm.37.1.62

Conejero-Suárez, M., Claver-Rabaz, F., Fernández-Echevarría, C., González-Silva, J., y Moreno-Arroyo, P. (2017). Toma de decisiones y rendimiento en las acciones de juego intermedias y finalistas en voleibol, en sets con diferente resultado. Psychology, Society y Education, 9(3), 459-468. DoI: https://doi. org/10.25115/psye.v9i3.1026

Fernández, J., Camerino, O., Anguera, M. y Jonsson, G. (2009). Identifying and analyzing the construction and effectiveness of offensive plays in basketball by using systematic observation. Behavior Research Methods, 41(3), 719- 730. DOI: https://doi.org/10.3758/brm.41.3.719

Fernández, M., Sánchez, C. Jiménez, F., Navarro, V., y Anguera, M. (2012). Sistema de codificación y análisis de la calidad del dato para una intervención inclusiva en Educación Física. Revista de Psicología del Deporte, 21(1), 67-73.

García, A. (2014). Perfil de rendimiento técnico-táctico en función de la categoría de competición en voleibol masculino (Tesis Doctoral). Universidad de Murcia, Murcia, España. Recuperado de http://hdl.handle.net/10201/37628

Gómez, M. (2014). Análisis del desempeño técnico-táctico de jugadoras de tenis de mesa de clase mundial, 1-3. Ministerio del Poder Popular para el Deporte Venezuela. DoI: http://doi.org/10.13140/2.1.4710.7206 
Gómez, M. (2017). La importancia del análisis notacional como tópico emergente en Ciencias del deporte. RICYDE. Revista Internacional de Ciencias del Deporte, 13(47), 1-4. DoI: http://doi.org/10.5232/ricyde2017.047ed

Hernández, R., Fernández, C. y Baptista, P. (2014). Selección de la muestra. Metodología de la Investigación, 6, 170-191. México: McGraw-Hill. Recuperado de http://euaem1.uaem.mx/bitstream/handle/123456789/2776/506_6. pdf? sequence $=1 \&$ is

Hughes, M., Hughes, M. y Behan, H. (2007). The evolution of computerised notational analysis through the example of racket sports. International Journal of Sports Science and Engineering, 10(31), 3-28. DoI: https://doi.org/10.21797/ ksme.2008.10.3.001

Kondrič, M., Zagatto, A. M., y Sekulić, D. (2013). The physiological demands of table tennis: a review. Journal of Sports Science \& Medicine, 12(3), 362-370. Recuperado de https://www.ncbi.nlm.nih.gov/pmc/articles/PMC3772576/

Malagoli, I., Di Michele, R., y Merni, F. (2014). A notational analysis of shot characteristics in top-level table tennis players. European journal of sport science, 14(4), 309-317. DOI: https://doi.org/10.1080/17461391.2013.819382

Martínez-Gallego, R. (2013). Análisis notacional en tenis. E-Coach. Área de docencia e investigación. Real Federación Española de Tenis, 12-16. Recuperado de https://www.researchgate.net/profile/Rafa_Martinez-Gallego/ publication/257815589_Analisis_notacional_en_tenis/links/00463525e 4486d1f83000000/Analisis-notacional-en-tenis.pdf

Martínez-Gallego, R. (2015). El análisis de la táctica en el tenis. E-Coach-Revista Electrónica del Técnico de Tenis, 24(8), 4-9. Recuperado de https://www. researchgate.net/profile/Rafa_Martinez-Gallego/publication/291814049_El_ analisis_de_la_tactica_en_el_tenis/links/56bc534708ae6521bfdf19e2.pdf

Medina J., García J., Morante, J., y Rodríguez, J. (2016). Análisis notacional de las acciones de juego realizadas por jugadoras de voleibol en competición universitaria. Sport TK-Revista Euroamericana de Ciencias del Deporte, 5(2), 113122. DOI: https://doi.org/10.6018/264751

Olmedilla, A., Ortega, E., Andreu, M., y Ortín, F. (2010). Programa de intervención psicológica en futbolistas: evaluación de habilidades psicológicas mediante el cprd. Revista de Psicología del Deporte, 19(2), 249-262. Recuperado de https://www.redalyc.org/pdf/2351/235116352012.pdf

Pradas, F., Pinilla, J., Quintas, A., y Castellar, C. (2014). Características de juego y estructura temporal en el tenis de mesa de alto nivel. Revista Internacional 
de Deportes Colectivos, 19, 5-16. Recuperado de: https://zaguan.unizar.es/record/60942/files/texto_completo.pdf

Pradas, F., Floría, P., González-Jurado, J., Carrasco, L., y Bataller, V. (2012). Desarrollo de una herramienta de observación para el análisis de la modalidad individual del tenis de mesa. Journal of Sport and Health Research, 4(3), 255-268. Recuperado de http://journalshr.com/papers/Vol\%204_N\%203/ V04_3_5.pdf

Pradas de la Fuente, F., Salvá Martínez, P., González Campos, G., y González Jurado, J. (2015). Análisis de los indicadores de rendimiento que definen el tenis de mesa moderno. Journal of Sport and Health Research, 7 (2), 149-162. Recuperado de https://idus.us.es/handle/11441/47601

Reid, M. (20-24 de Octubre de 2011). Moneyball for tennis. Men ITF Worldwide Coaches Conference, Egypt.

Reyes, M., Raimundi, M. J. y Gomez, L. (2012). Programa de Entrenamiento en Habilidades Psicológicas en jugadoras de voleibol de alto rendimiento. Cuadernos de Psicología del Deporte, 12(1), 9-16. DoI: https://doi.org/10.4321/ s1578-84232012000100001

Rodríguez-Salazar, M., y Montoya, J. (2006). Entrenamiento en el mantenimiento de la atención en deportistas y su efectividad en el rendimiento. Acta Colombiana de Psicología, 9(1), 99-112. Recuperado de https://www.redalyc. org/pdf/798/79890109.pdf

Roque, J. I. A., e Iturriaga, F. M. A. (2007). Utilización estratégica del espacio como indicador de rendimiento en el frontenis olímpico masculino. European Journal of Human Movement, 19, 77-95. Recuperado de https://dialnet.unirioja.es/servlet/articulo? codigo $=3322700$

Roque, J., e Iturriaga, F. (2011). Análisis notacional informatizado del rendimiento del saque en frontenis olímpico. Revista Internacional de Medicina y Ciencias de la Actividad Física y del Deporte/International Journal of Medicine and Science of Physical Activity and Sport, 11(42), 421-438. DoI: https://doi. org/10.5232/ricyde2008.01005

Sáez-Morales, G., Ruano-Anoceto, A., Gutiérrez-Pairol, M., y Águila-Crespo, M. (2018). ¿Cómo evaluar el rendimiento técnico-táctico durante la competición del tenis de mesa? Lecturas: Educación Física y Deportes, 22(237), 16-22. Recuperado de http://dspace.uclv.edu.cu/handle/123456789/12382

Sanz, D., y Terroba, A. (2012). Aplicación de las nuevas tecnologías al análisis de la táctica en el tenis. Coaching and Sports, Science Review, 56(20), 23-35. 
Recuperado de http://www.miguelcrespo.net/ITF\%20CSSR \%20E/ITF\%20 CSSR \%2056\%20Abr\%202012.pdf\#page $=23$

Takeuchi, T., Kobayashi, Y., Hiruta, S., y Yuza, N. (2002). The effect of the 40mm diameter ball on table tennis rallies by elite players. International Journal of Table Tennis Science, 5, 267-277. Recuperado de https://www.ittfeducation. com/effect-40mm-diameter-ball-table-tennis-rallies-elite-players/

Varas Caro, I., y Gómez Ruano, M. (2016). Análisis notacional en jugadores de tenis de élite en función de las variables contextuales-G-SE. Kronos, 15(1), 1-12. Recuperado de https://journal.onlineeducation.center/api-oas/v1/articles/ sa-c57cfb2727bc70/export-pdf

Weinberg, R., y Gould, D. (2010). Fundamentos de psicología psicología del deporte y del ejercicio físico físico/Fundamentals of Sport Psychology and Physical Exercise. Madrid: Editorial Médica Panamericana. 
